\title{
Lecithin and phytosterols-based mixtures as hybrid structuring agents in different organic phases
}

\author{
Paula K. Okuro ${ }^{\mathrm{a}}$, Antonio A. Malfatti-Gasperini ${ }^{\mathrm{b}}$, António A. Vicente ${ }^{\mathrm{c}}$, Rosiane L. Cunha ${ }^{\mathrm{a}, *}$ \\ a Department of Food Engineering, School of Food Engineering, University of Campinas, 13083-862 Campinas, São Paulo, Brazil \\ b Brazilian Synchrotron Light Laboratory, CNPEM, PO BOX 6192, 13.083-970 Campinas, São Paulo, Brazil \\ ${ }^{\mathrm{c}}$ Centre of Biological Engineering, University of Minho, Campus de Gualtar, 4710-057 Braga, Portugal
}

\section{A R T I C L E I N F O}

\section{Keywords:}

Gamma-oryzanol

Beta-sitosterol

Oleogel

Fat replacer

\begin{abstract}
A B S T R A C T
In this study the effect of lecithin (L) addition and solvent quality in a well-established oleogel system formed by $\beta$-sitosterol and $\gamma$-oryzanol (BG) was investigated. Medium chain triglycerides (MCT) and sunflower oil (SFO) were used as triglycerides and hexadecane (HEX) as a model of linear hydrocarbon. Lecithin was proposed due to its natural and versatile properties, showing different functionalities such as emulsifier and co-oleogelator. A study based on hierarchical organization of structured oil was performed applying techniques for bulk, meso and nanoscale. Self-sustained structures could no longer be observed after $40 \mathrm{wt} \%$ of BG replacement by lecithin. Small-angle X-ray scattering showed that the formed nanostructures (building blocks) were dependent on type of solvent and BG:L ratio in the mixture of oleogelators. Differential scanning calorimetry showed that stability against temperature was improved decreasing the polarity of the oil, and a time-dependent self-assembly of hybrid systems was observed from thermal and rheological measurements. Microscopy images exhibited changes on typical fibril aggregation of BG as lecithin was added, which promoted to a certain extent the suppression of ribbons. Oscillatory shear and uniaxial compression measurements were influenced by BG:L ratio and solvent mainly at higher lecithin amount. The combination of BG and MCT appeared to be the most affected by lecithin incorporation whereas SFO rendered harder oleogels. These results could contribute to understand the role of both lecithin and solvent type influencing the host oleogelator structure. It was hypothesized that intermolecular BG complex formation is hindered by lecithin, besides this phospholipid also might coexist as a different phase, causing structural changes in the gel network. Addressing the role of co-oleogelator it can provide the opportunity to tune soft materials with adjusted properties.
\end{abstract}

\section{Introduction}

The investigation of alternative routes for oil structuring have drawn a challenge for industry and researchers since texture of solid and semi-solid fats plays a key role on organoleptic and functional properties of food products. In this sense, oleogels have been studied as a promising system which can be formed by a diverse supramolecular ordering. Such diversity of mesoscale structures can lead to varied mechanical attributes, providing different semi-solid bulk phases (Patel, 2017; Patel \& Dewettinck, 2016; Terech \& Weiss, 1997).

Oleogels have been applied in many fields, albeit relatively few structuring agents can be considered food grade. In addition, other issues should be considered regarding food-related applications such as the replacement of hardstocks by healthful alternatives as plant-derived liquid oils aiming the replacement of saturated and mainly trans fatty acids, and the concerns involving sustainability of palm oil production
(Bot \& Agterof, 2006; Gravelle, Blach, Weiss, Barbut, \& Marangoni, 2017). Therefore, ingredients engineering may be applied to design suitable alternatives that could fulfil all the requirements, matching both technological functionality and nutritional features. However, the designing of an efficient gelator-solvent combination is not an easy task, and oleogel systems usually are serendipitous findings (Bot \& Agterof, 2006).

Oleogels can be formed from a single component or a mixture of different structuring agents (Bot, Den Adel, \& Rogers, 2008; Patel \& Dewettinck, 2016; Patel, 2017). Due to complex hierarchical structure of fats, it seems that single oleogelators systems are not likely to mimic and approach diverse functional properties of fats (Gravelle et al., 2017). The concept of multi component supramolecular gels offers the prospects to tailor gel characteristics, modifying functional properties for a target application by changing the ratio of the different components. Oleogelators mixtures may be classified in three main classes.

\footnotetext{
* Corresponding author.

E-mail address: rosiane@unicamp.br (R.L. Cunha).
} 
The first one requires all the added components to achieve the gelation, that is, neither of the single components work effectively as oleogelators on them own. The second considers that oleogelators may either co-assemble or self-sort into distinct assemblies. The third class consists of one (or more) oleogelator(s) and one (or more) non-gelling additive (s) and the latter can affect the assembly process of main oleogelator influencing the gel properties (Buerkle \& Rowan, 2012). Multicomponent gels can open up the possibility to optimize the functionality by altering the modulation of the self-assembly responsible for gel formation (Patel, 2017). Hybrid systems have been studied evaluating combinations such as fatty acids with fatty alcohols (Blach et al., 2016), $\gamma$-oryzanol with $\beta$-sitosterol (Bot \& Agterof, 2006; Bot, Adel, \& Roijers, 2008; Bot, den Adel, Roijers, \& Regkos, 2009), monoglycerides with phytosterols (Bin Sintang et al., 2017), lecithin with sorbitan tri-stearate (Pernetti, van Malssen, Kalnin, \& Flöter, 2007), lecithin with tocopherol (Nikiforidis \& Scholten, 2014), oleic acid with sodium oleate (Nikiforidis, Gilbert, \& Scholten, 2015) and ethylcellulose and stearyl alcohol/stearic acid (Gravelle et al., 2017).

Mixed component oleogels formed by $\beta$-sitosterol and $\gamma$-oryzanol are one of the most investigated systems (Bot \& Agterof, 2006). As single ingredients both components are able to form crystalline particles in vegetable oils but neither of them is capable of effectively entrap the liquid organic phase (Bot et al., 2008). However, when mixed at proper ratios, they co-assemble/co-crystallize to form fibril aggregates. As a result of suitable concentration of components, the primary nanoscale tubules may aggregate to form a 3-D network that can physically entrap the liquid oil through capillary forces to form a viscoelastic gel over macroscopic length scales (Alhasawi \& Rogers, 2013; Sawalha et al., 2012, 2015). An advantage in use plant sterol(ester)s is related to their composition with absence of saturated fatty acids, which usually are predominant in the crystallization of the most of other systems (Bot et al., 2008). $\beta$-sitosterol is a minor component found in vegetable oils, including soybean oil, while $\gamma$-oryzanol is present in trace amounts in rice bran oil, and it is comprised of ester of ferulic acid with a range of plant sterols (Xu \& Godber, 1999; Bonsdorff-nikander, Karjalainen, Rantanen, \& Christiansen, 2003). The combinations of $\gamma$-oryzanol with $\beta$-sitosterol or related phytosterols are based on chemical similarity regarding the presence of a ring or a hydroxyl group (Bot \& Agterof, 2006).

Lecithins have been widespreadly used as surfactant agent and crystal habit modifier (changing crystal morphology) depending on food matrix, physical and colloidal state (Nikiforidis \& Scholten, 2014). However, lecithin oleogels have limited potential for food application due to their sensitivity to water (Kumar \& Katare, 2005). Lecithin has been applied as co-oleogelator prone to change the self-assembly of host structuring agent, leading to an improvement of gelation in mixture with sorbitan tristearate (Pernetti et al., 2007), sucrose esters (Bin Sintang et al., 2017) and $\alpha$-tocopherol (Nikiforidis \& Scholten, 2014), but hindering the network formation in a blend with 12-hydroxystearic acid (Tamura \& Ichikawa, 1997). Some researchers have been reporting positive outcomes from lecithin inclusion in oleogelators mixture such as improvement in gelation, either by accessing the gel formation (Pernetti et al., 2007; Han et al., 2013) or indirectly by acting as a nongelling additive (Patel, 2017;). $\beta$-sitosterol, $\gamma$-oryzanol and lecithin are considered GRAS ingredients besides their positive healthy-related effects. In this study, a combination of a well-known pair of oleogelators ( $\beta$-sitosterol and $\gamma$-oryzanol) with lecithin combined with different solvents was investigated.

Solvent quality might be considered to predict solvent-gelator interactions since it can mediate oleogel formation (Gao, Wu, \& Rogers, 2012), and affect gel properties (Gravelle, Davidovich-Pinhas, Zetzl, Barbut, \& Marangoni, 2016). The solvent polarity is mainly governed by chain length of fatty acids, proportion between saturated and unsaturated fatty acids, and also presence of functional groups (either polar and non-polar) (Gravelle et al., 2016).

In view of that, different solvents were tested with a mixture of natural-based gelators to obtain a self-standing material with ability to retain oil. The purpose is to elucidate the effect of lecithin addition and solvent quality in a plant sterol(ester)s-based oleogel. It was hypothesized that lecithin might be able to change not only the molecular assembly of BG but the mesoscale organization providing unique bulk gelled systems with different elastic characteristics to be aligned with diverse applications.

\section{Materials and methods}

\subsection{Materials}

$\beta$-sitosterol (B) was purchased from Sigma-Aldrich (85451-St. Louis, USA) with purity $>70 \%$ (non-esterified form, residual $\beta$-sitostanol, campesterol and campestanol). The $\gamma$-oryzanol (G) 99\% purity was kindly received as gift from Tsuno Rice Fine Chemicals (Wakayama, Japan). The ingredients were used without further purification. Soybean lecithin (L) Phospholipon ${ }^{\circledR}$ 90G (96.9 wt\% phosphatidylcholine, $1.1 \mathrm{wt} \%$ lysophosphatidylcholine) was supplied from Lipoid $\mathrm{GmbH}$ (Ludwigshafen, Germany). The fatty acids composition present in hydrophobic tails of lecithin was approximately $62 \%$ linoleic acid (C18:2), 15\% palmitic acid (C16:0), 12\% oleic acid (C18:1), 5\% linolenic acid (C18:3) and 3\% stearic acid (C18:0). Hexadecane (HEX) $\left(\mathrm{C}_{16} \mathrm{H}_{34}\right)$ (Sigma Aldrich, USA), Delios V (MCT) (Cognis, Germany) and regular sunflower oil (SFO) (Bunge Alimentos S.A., Brazil) were investigated as organic phases. Hexadecane is a linear chain hydrocarbon (average molecular weight, $\mathrm{MW}=226.4 \mathrm{~g} \mathrm{~mol}^{-1}$ ), and usually studied as a model owing to its low polarity and viscosity ( $3 \mathrm{mPas}$ ). MCT is a medium-chain triglyceride ( $\mathrm{MW}=554 \mathrm{~g} \mathrm{~mol}^{-1}$ ), rich in caprylic (C8:0) and capric glycerides (C10:0) showing viscosity of $24 \mathrm{mPa}$. SFO is mainly comprised of linoleic (C18:2), oleic (C18:1), palmitic (C16:0) and $\alpha$-linolenic $(\mathrm{C} 18: 3)$ acids, $\left(\mathrm{MW}=879 \mathrm{~g} \mathrm{~mol}^{-1}\right.$ ) showing high viscosity ( $56 \mathrm{mPas}$ ). The polarity of those three solvents is increasing for HEX $<$ SFO < MCT.

\subsection{Gel preparation}

$\beta$-sitosterol and $\gamma$-oryzanol mixtures (BG) at fixed molar ratio of 1:1 or equivalent $2: 3$ on weight basis and lecithin (L) were properly weighted to scan different ratios BG:L (100:0; 80:20; 60:40; 50:50; 40:60; 20:80; 0:100), named as BG100; BG80L20; BG60L40; BG50L50; BG40L60; BG20L80; L100, respectively. The total oleogelator amount was kept fixed (20 wt\%) (Bot \& Agterof, 2006; Sawalha et al., 2012). Afterwards BG, BG + L and L as mono or multicomponent structuring agents were homogenised with the different liquid solvents (HEX, SFO or MCT) by heating at $90 \pm 5{ }^{\circ} \mathrm{C}$ and applying agitation (200-300 rpm) using a magnetic stirrer until all oleogelators were fully dissolved and the content became visually clear and homogeneous. Samples were cooling down under mild agitation at room temperature. All analyses were performed with samples stored at least $48 \mathrm{~h}$ at room temperature after their preparation.

\subsection{Small-angle $X$-ray scattering}

Nanoscopic structural information of oleogels was obtained from Small-Angle X-ray Scattering (SAXS) measurements which were performed at the SAXS 1 beamline at Brazilian Synchrotron Light Laboratory (LNLS/CNPEM, Campinas, Brazil). Experiments were performed using an $8 \mathrm{keV}$ beam energy $(\lambda=0.155 \mathrm{~nm})$ and a sample-todetector distance of $1010.5 \mathrm{~mm}$. The data are shown as plots of the SAXS intensity, $I(Q)$, as a function of the modulus of the scattering vector $Q=4 \pi \sin (\theta) / \lambda$, where $2 \theta$ is the scattering angle and $\lambda$ is the beam wavelength. Each SAXS pattern corresponds to a data collection time of 50 or $100 \mathrm{~s}$. All $I(Q)$ data were corrected for a background scattering from Kapton tape for gel-like samples, and mica window for the liquid holder device. The scattering data are presented after 
subtraction of solvent contribution. To understand how the building blocks of oleogel are affected by temperature, the X-ray scattering profile was measured from 10 to $66^{\circ} \mathrm{C}$ each $2{ }^{\circ} \mathrm{C}$ for BG60L40 with SFO. Temperature was controlled by a water bath connected to the sample holder.

\subsection{Optical properties-opacity}

Oleogels were structured as a thin layer $(3 \mathrm{~mm})$ in a glass petri dish $(\varnothing: 50 \mathrm{~mm})$ and they were subjected to opacity analysis using a colorimeter (UltraScan VIS, HunterLab, Virginia, EUA) operating in transmission mode. The CIELab and illuminant D65 (daylight) (Hunterlab, 1996) was used. The haze (opacity) parameter was directly classified by the EasyMatch QC software. The experiments were performed at least in triplicate and the results are presented as mean values.

\subsection{Differential scanning calorimetry}

The thermal behavior of oleogels was investigated with differential scanning calorimetry (DSC) using a thermal analyser Modulated DSC2920 (TA Instruments, New Castle, Delaware, USA). Hermetic Aluminium pans containing around $10 \mathrm{mg}$ of oleogel sample were equilibrated at $25^{\circ} \mathrm{C}$, heated up to $110^{\circ} \mathrm{C}$ and maintained at this temperature for $5 \mathrm{~min}$, and then cooled down to $0^{\circ} \mathrm{C}$ and kept isothermally at $0^{\circ} \mathrm{C}$ during $15 \mathrm{~min}$. After that, samples were heated again to $110^{\circ} \mathrm{C}$. The heating and cooling rates were 10 and $5{ }^{\circ} \mathrm{C} / \mathrm{min}$, respectively. Data were processed by the TA Universal Analysis software. To have some insights about the kinetic involved in oleogel formation in the presence of lecithin, pans were subjected to a second scan after storage at room temperature for $48 \mathrm{~h}$. Information about the structure evolution over time was accessed by the comparison between the first melting cycle of the first run with the first heating of the second run (after storage). Gels produced with HEX were cooled until $20^{\circ} \mathrm{C}$ (instead of $0{ }^{\circ} \mathrm{C}$ ) since the melting profile of hexadecane shows a well-defined endothermic peak at around $19.22^{\circ} \mathrm{C}$ and a phase-change enthalpy of $227.2 \mathrm{~J} \mathrm{~g}^{-1}$ (Yin, Liu, Geng, Zhang, \& Zhang, 2015).

\subsection{Optical microscopy}

The microstructure of oleogels was observed using an optical microscope Olympus BX51TF (Olympus, Japan) equipped with a digital camera. A drop of melted sample collected just after gel preparation, was placed in a glass slide and a cover slip was gentle put on top. Prepared slides were examined after $48 \mathrm{~h}$ of storage at room temperature.

\subsection{Rheological measurements}

Linear viscoelastic region (LVR) of oleogels was defined by small amplitude dynamic measurement using a stress-controlled rheometer Physica MCR 301 (Anton Paar, Graz, Austria) equipped with a Peltier system and water bath (Julabo, Seelbach, Germany) for temperature control. A strain sweep was performed from 0.01 to $10 \%$ at a frequency of $1.0 \mathrm{~Hz}$. The experiment was performed using $50 \mathrm{~mm}$ sandblasted parallel-plate geometry, with a roughness of $5-7 \mu \mathrm{m}$ and a gap set at $1000 \mu \mathrm{m}$.

The rheological measurement was performed following the steps: (a) temperature ramp from $25^{\circ} \mathrm{C}$ until $90^{\circ} \mathrm{C}$; (b) isothermal for $15 \mathrm{~min}$ at $90^{\circ} \mathrm{C}$; (c) temperature ramp from $90^{\circ} \mathrm{C}$ until $5{ }^{\circ} \mathrm{C}$ for MCT and SFO, and $20^{\circ} \mathrm{C}$ for HEX; (d) $40 \mathrm{~min}$ at $5^{\circ} \mathrm{C}$ (for MCT and SFO) and $20^{\circ} \mathrm{C}$ for HEX; and (e) frequency sweep from 0.01 to $10 \mathrm{~Hz}$ at $20^{\circ} \mathrm{C}$. The elastic component $\left(G^{\prime}\right)$ and viscous component $\left(G^{\prime \prime}\right)$ were recorded.

All the measurements were performed at constant strain of $0.1 \%$ and steps a, b, c, and d were evaluated at $1 \mathrm{~Hz}$. When hexadecane was used as solvent the applied temperature to rebuild the gel structure was $20^{\circ} \mathrm{C}$ since the melting temperature of hexadecane is around $18-19^{\circ} \mathrm{C}$, in contrast with MCT and SFO where the isothermal conditions for structure development was $5{ }^{\circ} \mathrm{C}$ during $30 \mathrm{~min}$.

\subsection{Large deformation properties: uniaxial compression}

Oleogels were structured in a cylindrical shape $(20 \mathrm{~mm}$ diameter $\times 20 \mathrm{~mm}$ height). These samples were subjected to uniaxial compression using a TA-XT-Plus Texture Analyser (Stable Microsystems Ltd., Surrey, UK) with an acrylic cylindrical plate $(80 \mathrm{~mm}$ diameter) lubricated with the corresponding solvent (HEX, SFO or MCT) minimizing friction between gel and probe. The samples were compressed at a speed of $1 \mathrm{~mm} / \mathrm{s}$ until $80 \%$ of its original height.

Hencky stress $\left(\sigma_{H}\right)$ and strain $\left(\varepsilon_{H}\right)$ were calculated from the forcedeformation data according to Eqs. (1) and (2), respectively:

$\sigma_{H}=F(t)\left[\frac{H(t)}{H_{0} A_{0}}\right]$

$\varepsilon_{H}=-\ln \left[\frac{H(t)}{H_{0}}\right]$

where $F(t)$ is the force at time $t, A_{0}$ and $H_{0}$ are the initial area and height of the sample, respectively, and $H(t)$ is the height at time $t$. The rupture properties were obtained from the first maximum point of the stress-strain curve. Stress $\left(\sigma_{R U P}\right)$ at rupture was used as an index of the gel hardness and the strain $\left(\varepsilon_{R U P}\right)$ at rupture related to the gel deformability. The Young's or elasticity modulus (E) was obtained from the slope of the initial linear viscoelastic region of the stress-strain curve $\left(0.01-0.02<\varepsilon_{H}<0.06\right)$.

\section{Results}

\subsection{Small-angle $X$-ray scattering}

The morphology and interactions of the building blocks which form network can be decisive to impart structure on oils. Efficient oleogelators form small and preferably non-spherical building blocks, providing a high specific surface area to form network connections (Bot, Veldhuizen, den Adel, \& Roijers, 2009). In this work, SAXS behaviour was addressed in a qualitative approach. The SAXS data of different ratios BG:L in HEX, SFO and MCT are shown in Fig. 1. The form factor for all BG100 was based on previous studies (Bot et al., 2008; Bot, den Adel, et al., 2009; Bot, Veldhuizen, et al., 2009). The consolidate building block model is described as a hollow cylinder appearing as thin tubules composed of $\beta$-sitosterol and $\gamma$-oryzanol. A more detailed description indicating a double-walled tubule composed of an inner and an outer shell containing the androsterol group of sitosterol or oryzanol and the ferulic acid moieties of oryzanol molecule is also reported on literature (Bot et al., 2012).

The scattering profiles of BG in HEX, SFO and MCT are presented in Fig. 1a(1), b(1) and c(1). They exhibit similar broad maxima located at $0.95 ; 1.90 ; 2.69 \mathrm{~nm}^{-1}$ for HEX, $0.96 ; 2.00 ; 2.75 \mathrm{~nm}^{-1}$ for SFO and $1.00 ; 1.99 ; 2.88 \mathrm{~nm}^{-1}$ for MCT, which indicates the predominance of similar structures regardless of solvent quality. These profiles are in agreement with the values reported for ternary mixtures by other groups (Bot et al., 2008; Sawalha et al., 2015). In addition, the depth and sharpness of minima indicates that the form factor is rather monodisperse, which is not valid for other samples containing lecithin (lines (2) to (6)) (Bot et al., 2008). A clear change in SAXS pattern takes place increasing lecithin amount in oleogelators mixture (Fig. 1a, b and c lines (2) to (6)). A qualitative difference among the scattering profiles was remarkable for both solvent chemistry and lecithin addition which might correlate with gelling ability. It is well known that the SAXS slope at low $Q$ gives information about the morphology of the building blocks. A $Q^{-1}$ slope suggests the formation of linear structures such as 

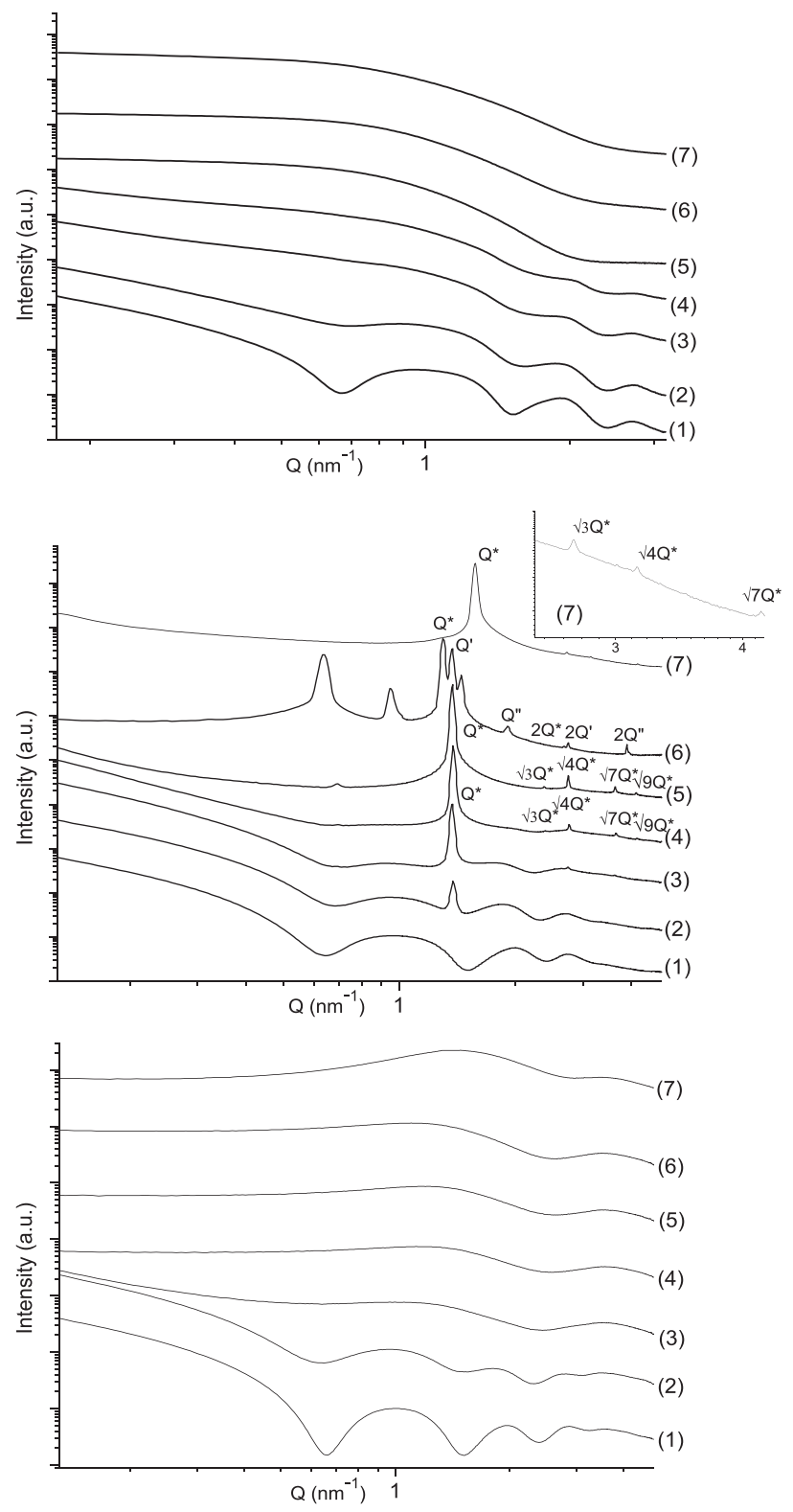

Fig. 1. Experimental X-ray scattered intensities, $I(Q)$, of $\beta$-sitosterol and $\gamma$-oryzanol (1), $\beta$-sitosterol, $\gamma$-oryzanol and lecithin mixtures (2-6) and lecithin (7) in hexadecane-HEX (a), sunflower oil-SFO (b) and medium chain triacylglycerol-MCT (c) as organic solvent. The curves were shifted vertically for better visualization.

tubules while a $Q^{O}$ slope (constant scattering) suggests micellar structures (Sawalha et al., 2015).

A change in $Q$ slope as a result of lecithin addition is clearly seen from all systems presented in Fig. 1. The change is more pronounced for MCT followed by HEX and SFO, respectively. At high BG concentration (curves 1, 2 and 3, mainly), the slope at low $Q$ suggests the formation of linear structures, e. g. the tubule structure (Bot et al., 2008, 2012; Bot, den Adel, et al., 2009). For MCT, the $Q^{-1}$ slope spams from curve 1 (BG100) to curve 3 (BG60L40), although the tubule form factor features (such as the minima located between 0.6 and $0.7 \mathrm{~nm}^{-1}$ ) are only present in curves 1 and 2. Similarly, for HEX, the $Q^{-1}$ slope are present in curves 1 to 4, although the tubule form factor features are almost absent in curve 4. For SFO, in the other hand, although the tubule form factor features are only present from curves 1 to 3 , the $Q^{-1}$ slope is present for all other curves with exception of curve 6 . For systems with higher amount of lecithin in HEX and MCT, the low $Q$ behaviour suggests that these systems are composed by (reverse) micelles. The maximum occurring near $Q=1.4$ to $1.8 \mathrm{~nm}^{-1}$ for MCT (position depending on lecithin fraction) suggests a small degree of aggregation of these micelles. Although there is no evident correlation peak in the case of the lecithinrich HEX systems, simulations performed by our group using an elongated micelle (worm-like micelle) model have shown that these systems also present a small degree of aggregation.

The modifications on SAXS patterns taking HEX as organic solvent as result of lecithin addition can be described as follow: From (1) to (2) is possible to point that lecithin addition slightly changes the cylinder structure (the hollow tubule), since the position of minima in the tubule form factor change places. In curves (3) and (4), the tubule form factor oscillations for high $Q$ values still can be seen; however, it is clear that lecithin addition changed this form factor. For curve (5), the tubule form factor oscillations disappear, and a micelle-like scattering pattern takes place. Although at low $Q$ the scattering seems to be constant for this system suggesting that it is composed by small micelles, simulations using a worm-like micellar system have shown that this kind of structure also can be present. The exact shape and size of these structures cannot be extracted from the SAXS curves due to the limited $Q$ range at low $Q$ accessed in the experiments.

Similar effects can be seen when taking MCT as organic solvent. Curve (2) also shows a slightly change in the tubule form factor, since the position and intensity ratio of maxima slightly changes, which can be interpreted as a modification in the tubule structure due to the inclusion of lecithin. The difference from HEX systems is that already for curve (3) $-40 \%$ of lecithin - is not possible to observe the intensity oscillations due to the tubule form factor. However, the tubule presence - or, most probably, another 1D structure such as an elongated micelle - can be identified due to the low $Q$ behaviour. From curve (4) to (7), the scattering patterns change to a (reverse) micellar system; these scattering patterns are quite similar, except for a maximum appearing in the range of 1.5 to $1.8 \mathrm{~nm}^{-1}$ (depending on lecithin fraction) which becomes more pronounced as the lecithin fraction increases. This maximum can be interpreted as a correlation peak that appears due to the micelle aggregation. For both MCT and HEX systems, the micelle form factor also slightly changes as the lecithin fraction increases; this effect, together with the absence of the tubule form factor oscillations are strong evidences that the BG molecules are part of the micelle structure, i.e. these molecules migrate from tubules to the micelles.

The SFO systems however, showed a more complex behaviour as the lecithin fraction increases. From curve (1) to (2) it is possible see that lecithin addition changes the tubule structure, since local minima of the form factor change places. Also, is possible to see that a strong peak appears near $Q=1.38 \mathrm{~nm}^{-1}$, due to a formation of a (most likely) lecithin-rich phase. In addition to this peak, no features from the scattering profile can be easily attributed to this new phase. From curve (2) to (3), both phases (tubule and lecithin-rich) still remain, although tubule phase decreases in intensity and changes its structure (since the form factor minima change positions and relative intensity of oscillations changes). The peak located at $Q=1.38 \mathrm{~nm}^{-1}$ from lecithin-rich phase increases in intensity, and it is possible to see two higher-order peaks which correspond to orders $\sqrt{ } 4$ and $\sqrt{ } 7$ with respect to the former peak, suggesting that the lecithin-rich phase is organized in a two-dimensional inverse hexagonal array. The peak corresponding to the ratio $\sqrt{ } 3$ can only be seen in curve (4), where the order $\sqrt{ } 9$ also can be seen. Curve (5) is similar to curve (4) in many aspects although the oscillations from tubule form factor have disappeared. A low intensity peak located at $Q=0.69 \mathrm{~nm}^{-1}$ also can be seen. In curve (6), a plenty of peaks appears. Observing the peak positions, intensities and widths, it is not possible to associate this curve to a single or to a two-phase system; at least three phases are necessary to describe all peaks. Curve (7), however, presents peaks at positions which correspond to a hexagonal phase (first peak position at $Q=1.586 \mathrm{~nm}^{-1}$ and at ratios of $1: \sqrt{ } 3: \sqrt{ } 4$ : 7 ). This phase, interestingly, does not appear in any other system composed by the mixture of lecithin and BG in SFO, suggesting that 

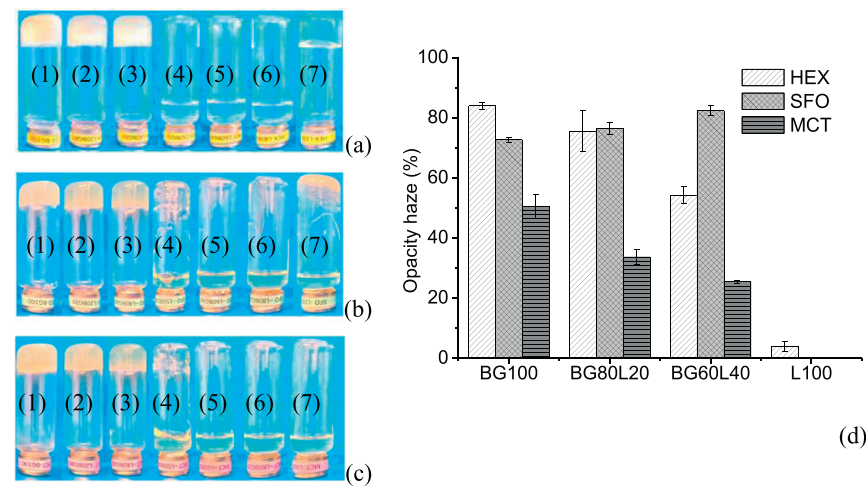

(d)

Fig. 2. Visual appearance of $\beta$-sitosterol/ $\gamma$-oryzanol (BG), lecithin (L) and BG:L oleogels in HEX (a), SFO (b) and MCT (c) (top to bottom) after $\sim 48 \mathrm{~h}$. The BG:L ratios ranged from 100:0 (1); 80:20 (2); 60:40 (3); $50: 50$ (4); 40:60 (5); 20:80 (6) and 0:100 (7). Opacity haze (\%) for oleogels produced with BG, L and BG:L with hexadecane, sunflower oil or medium chain triacylglycerol (d).

lecithin and BG tend to mix in SFO and create stable phases, while pure lecithin-SFO system tend to form elongated micelles organized in a hexagonal array. The huge difference in intensity between the first and other order peaks can be justified by both hexagonal package disorder and micelle form factor (most probably), since form factor modulates peak intensities. This is also valid for other lecithin fraction systems (curves (2) to (6)).

These results showed that lecithin affects the fibrous structure and fibrous aggregation of $\beta$-sitosterol and $\gamma$-oryzanol, since lecithin is promoting changes in BG system at nanoscale at different levels depending on BG:L mixture ratio.

\subsection{Gel formation and appearance}

The effect of different ratios between $\beta$-sitosterol $/ \gamma$-oryzanol and lecithin (BG100, BG80L20, BG60L40, BG50L50, BG40L60, BG20L80 and L100) was evaluated for a fixed B:G molar ratio. Fig. 2(a, b and c) illustrates the systems formed from those combinations in HEX, SFO and MCT, respectively. Gel formation was observed for all solvents combined with the pair $\beta$-sitosterol/ $\gamma$-oryzanol (BG100), which is in agreement with the SAXS patterns showing the presence of similar building blocks regardless solvent type. Otherwise, the control with lecithin (L100) yielded a gel-like structure only with hexadecane (Fig. 2a:7), while some phase separation could be observed with SFO (Fig. 2b:7) and a total solubilisation of this phospholipid in MCT (Fig. 2c:7). Lecithin exhibits a rich and varied phase behavior, especially in ternary or quaternary systems (Pernetti et al., 2007), but the lower polarity of hexadecane seems to exert a key role to the lecithin self-assembly and gel formation. Regarding the oleogelator mixture, the replacement of BG by until $40 \mathrm{wt} \%$ still formed gel and beyond that, the network could no longer self-standing after the tubes inversion (Fig. 2). The systems which had no oil structuration appeared as a clear solution as lecithin was included ( $>50 \mathrm{wt} \%$ ), with the exception of L100 in SFO (Fig. 2b:7).

The opacity values of the samples are shown in Fig. 2d. The interaction between sitosterol and oryzanol promoted the formation of opaque gels, which was caused by the limited number of stable nuclei. Thus, the system would have less growth sites for the crystalline materials to develop, driving the crystals growth in shape of long thick crystalline aggregates that diffract light and result in opaque gels (Rogers, Bot, Lam, Pedersen, \& May, 2010). More opaque gels were produced with HEX in the absence of lecithin (BG100) followed by SFO and MCT. However, the opacity decreased for HEX and MCT with BG reduction and $\mathrm{L}$ addition, but increased for SFO.

As opposed to these results, the tendency of SFO oleogel to became more opaque might be related with the formation of new phases as
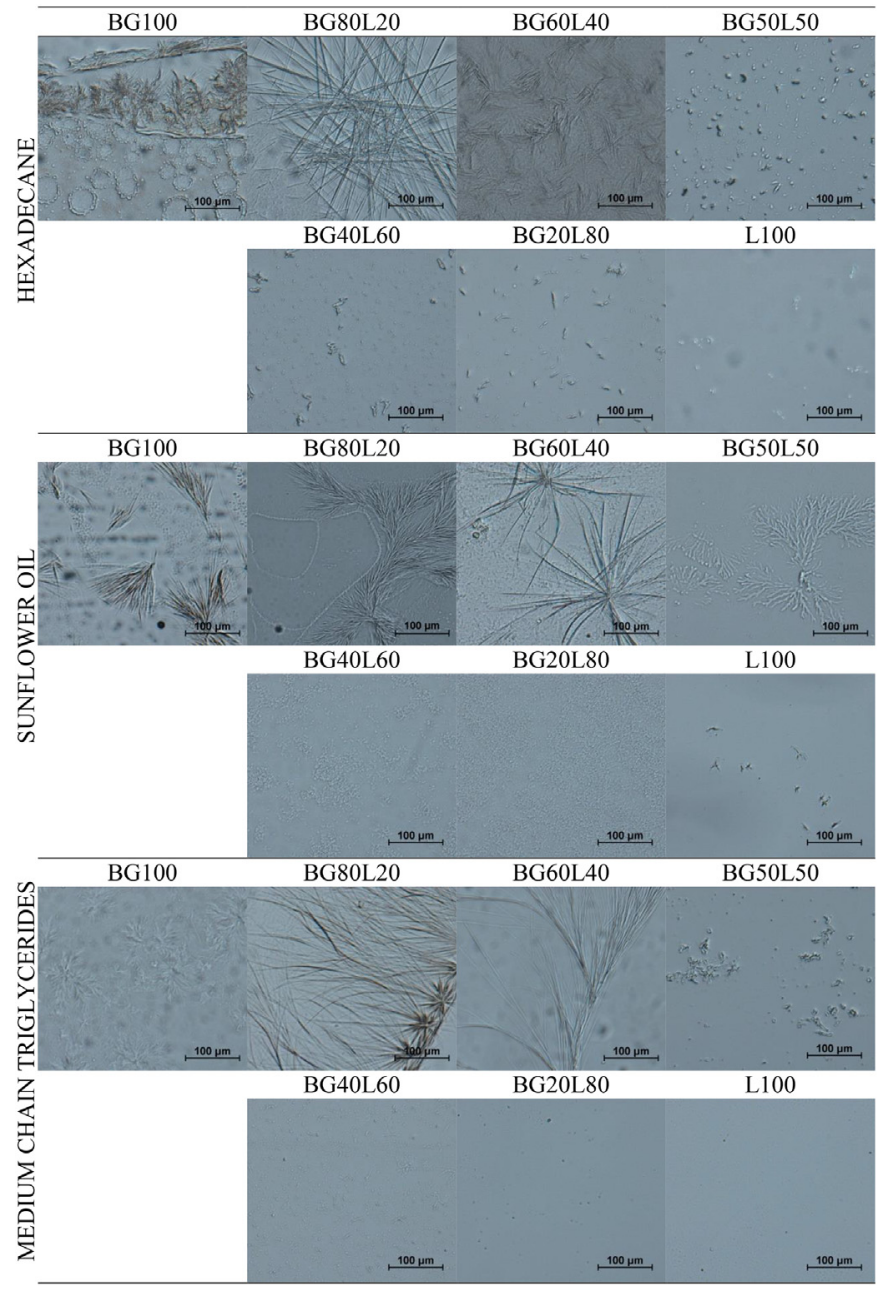

Fig. 3. Optical microscopy for oleogels produced with $\beta$-sitosterol $/ \gamma$-oryzanol (BG) + Lecithin (L) mixture in hexadecane, sunflower oil and medium chain triglycerides, respectively.

reported by SAXS analysis (Fig. 1b), which is not observed neither for HEX nor MCT. Another characteristic that could be observed through gel formation was the time required to samples forming gel-like structures. After the dissolution of $\beta$-sitosterol and $\gamma$-oryzanol the system could remain liquid during determined period of time for MCT and SFO, whereas for HEX a gelation was observed just after samples cooling. It noteworthy that the correlation between gelling behaviour with the tubules presence (SAXS), whether the presence of tubules indicates the formation or not of oleogels, seems to be not strictly true since the tubule formation would also occur in fluid mixtures (Sawalha et al., 2015).

\subsection{Microstructure}

Fig. 3 depicts the microstructure of the oleogels formed with BG, L and BG:L mixture in HEX, MCT and SFO. The morphology of BG oleogel has been described by different research groups as fibrous microstructure that further is organized into bundles of fibrils (Bot \& Agterof, 2006; Bot et al., 2008; Matheson, Koutsos, Dalkas, Euston, \& Clegg, 2017). This typical fibrillar structure was observed for BG oleogels in all solvents (BG100). Presence of fibrils was even observed after the replacement of BG by 20 or $40 \mathrm{wt} \%$ by lecithin. From $50 \mathrm{wt} \%$ replacement of BG was no longer possible to observe the fibrils formation in the samples with the exception of SFO. It turns to the light that gel like structure is related to the fibrils presence, but the shape and feature of this fibril-like structure is affected by both solvent quality and L 

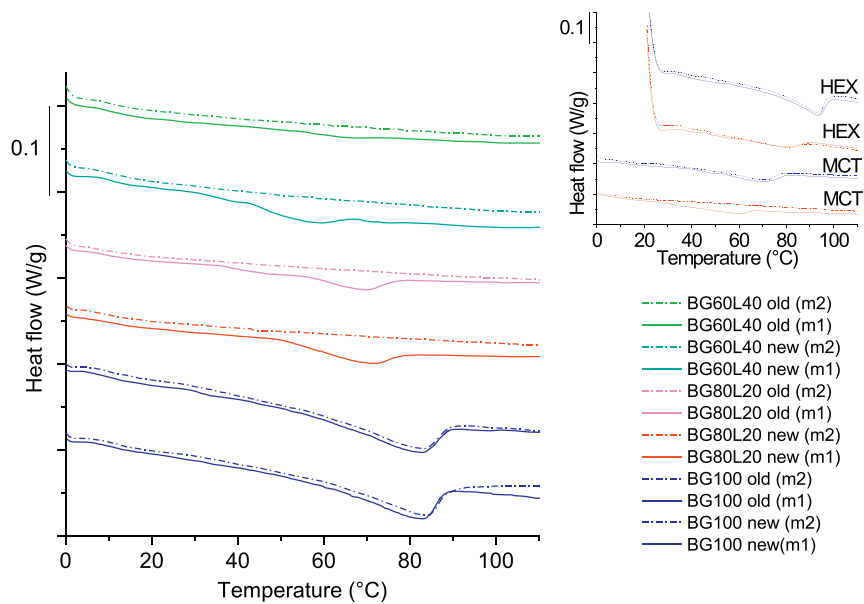

Fig. 4. Representative DSC melting profiles from the first (solid line: $\mathrm{m} 1$ ) and second (dashed line: $\mathrm{m} 2$ ) melting cycle of the same scan for oleogels produced with SFO (BG100, BG80L20 and BG60L40). Effect of storage was indicated with the words new and old, corresponding to the samples before and after storage, respectively. The inset figure shows the melting profile for oleogels produced with HEX and MCT as solvent for BG100 (blue line) and BG80L20 (red line) formulations. (For interpretation of the references to colour in this figure legend, the reader is referred to the web version of this article.)

addition. Akin to the case of interaction between lecithin and 12-hydroxystearic acid, the phospholipid changed the fibrous network of the main oleogelator. Lecithin seems to disturb the continuity of BG crystalline network resulting further in a different bulk material. Above replacement of $50 \mathrm{wt} \%$ of lecithin in the mixture the micrographs showed no fibrous structure formation, which would explain the transparency of non-gelled liquid systems (Fig. 2a,b,c).

\subsection{Thermal analysis}

\subsubsection{Differential scanning calorimetry (DSC)}

The thermal behavior of the BG:L oleogels were studied to characterize the impact of lecithin addition on melting properties of sterol (ester)s' crystal network. Fig. 4 exhibits representative melting profiles of SFO oleogels (BG100, BG80L20 and BG60L40) since no peaks were observed above $50 \mathrm{wt} \%$ of lecithin. The thermal response of oleogels produced with SFO was accessed by comparing the first (m1) and second (m2) melting (solid and dashed line) as also performed to HEX and MCT (BG100 and BG80L20) presented in the inset figure. In addition, the thermogram of the same pan was collected before and after storage, indicated by words "new" and "old", corresponding to scans before and after storage respectively. The second scanning of the same pan after storage ("old") was carried out since it was observed a different melting profile in the second heating cycle (after erase crystal memory), comparing solid and dashed lines ( $\mathrm{m} 1$ and $\mathrm{m} 2$ ), which was associated to a delayed crystallization caused by lecithin addition. Indeed, the second melting (dashed line) was not equivalent with first melting (solid line) for BG80L20 and BG60L40 oleogels (Fig. $4 \mathrm{~m} 1$ and $\mathrm{m} 2$ ), but after storage the melting peak reappears (Fig. 4 old: $\mathrm{m} 1$ ) indicating the development of network upon storage. In the other hand, BG100 samples showed no difference comparing first and second melting cycles (solid and dashed line) or storage effect (new or old). Moreover, the inset figure of Fig. 4 showed that MCT presented the same pattern of SFO, while for HEX, lecithin acted in lower extent disturbing BG network formation overlaying first and second melting cycles (solid and dashed line). These results indicate that the mixture between L and BG promote a change in thermal behavior and show that the crystalline structure takes longer time to be formed with lecithin mainly for SFO and MCT. Besides that, due to the solvent-gelator interaction, more polar solvents, as SFO and MCT, are reported to be more likely to dissolve oleogelators molecules hindering gelation or crystallization (Lan \& Rogers, 2015).

Endotherms resulting from $\mathrm{L}$ inclusion showed progressively lower peak melting temperature as crystalline BG content was decreased, since the total oleogelator concentration was fixed for preparation of hybrid oleogels, as can be seen in Fig. 5. There was a continuous depression in both onset and peak melting temperature and the shape of peak broadened as the concentration of phospholipid increased.

\subsubsection{Temperature assisted SAXS}

Temperature assisted in-situ small-angle X-ray scattering (SAXS) was performed to examine structural changes of BG:L oleogel from 10 to $66^{\circ} \mathrm{C}$. Fig. 6 shows the SAXS intensity for BG60L40 oleogel in SFO following sequential heating. SAXS measurements have shown that the temperature influenced the self-assembly of hybrid oleogelator mixture. The sharp peak initially in $1.43 \mathrm{~nm}^{-1}$ at $10^{\circ} \mathrm{C}$ was slightly displaced to higher $Q$ with the increase of temperature, reaching $1.46 \mathrm{~nm}^{-1}$ at $38^{\circ} \mathrm{C}$. As discussed previously, this peak is related to a lecithin-rich phase composed by reverse micelles which is arranged in a hexagonal array. At $38^{\circ} \mathrm{C}$, the peak cannot be observed anymore, showing a disruption of the hexagonal array. This disruption might be related with the melting peak found in the DSC results (onset and peak melting temperatures of $43 \pm 1$ and $53 \pm 6{ }^{\circ} \mathrm{C}$, respectively). Other features of the scattering intensity, such as the oscillations due to the tubule form factor and the low- $Q$ scattering intensity change with temperature. The depth and sharp of the form factor minima gets smoother with increase of temperature, probably due to the softening of the tubule structure. Probably the decrease of the low- $Q$ scattering intensity with temperature is also related to the tubule softening.
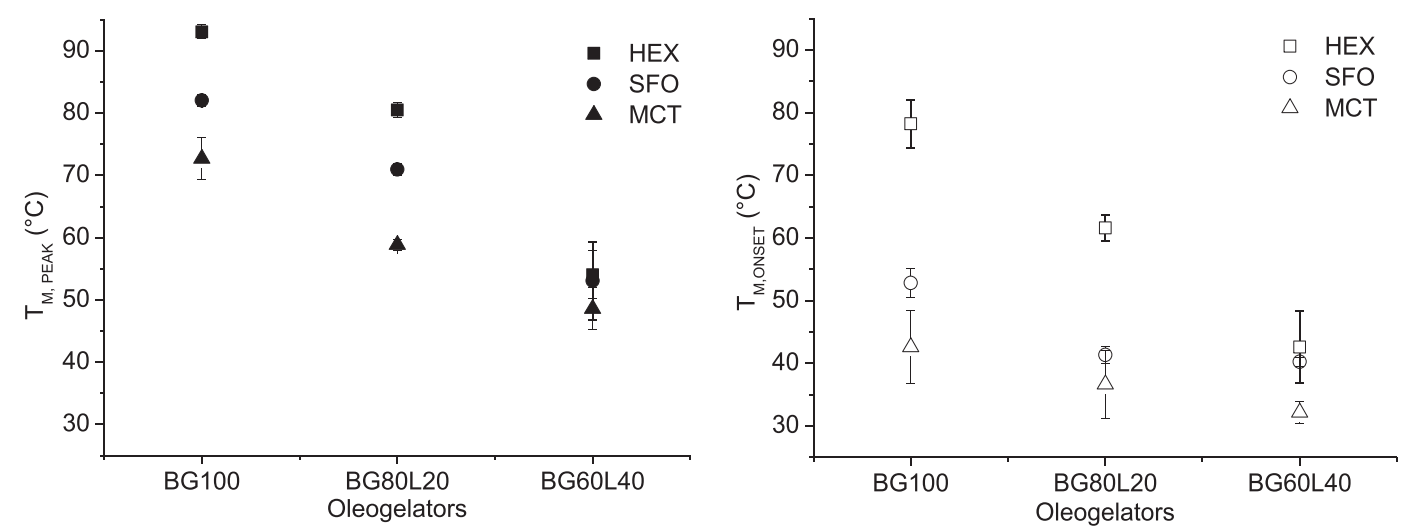

Fig. 5. Peak (solid symbols) and onset (open symbols) melting temperatures obtained from the first melting cycle for BG and BG:L oleogels as function of BG:L ratio $(100: 0,80: 20$ and 60:40). 


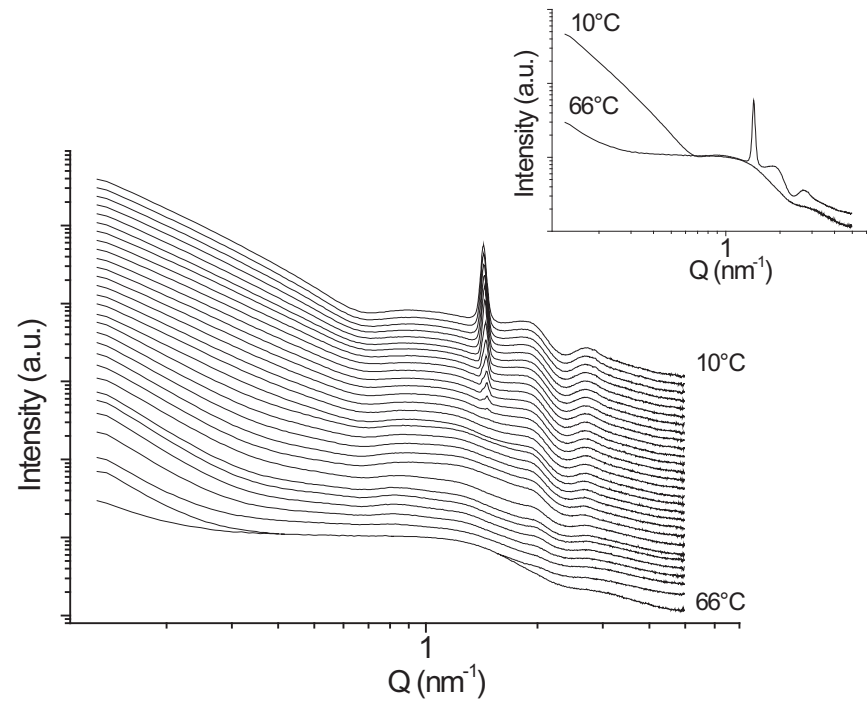

Fig. 6. Small-Angle X-ray scattering intensity of SFO oleogel (BG60L40) at different temperatures ranging from 10 to $66^{\circ} \mathrm{C}$.

\subsection{Response of the hybrid oleogels to small and large deformations}

\subsubsection{Rheological measurements}

Fig. 7 shows the effect of lecithin in non-isothermal and isothermal rheological measurements. The temperature-dependent response of elastic $\left(G^{\prime}\right)$ and viscous $\left(G^{\prime \prime}\right)$ moduli could be observed (Fig. 7a, c and e), showing that lecithin addition increased the thermal sensitivity of oleogels. Oleogels exhibited a reduction in both $G^{\prime}$ and $G^{\prime \prime}$ values during heating. This decay intensity (or thermal sensitivity) was HEX $<$ SFO < MCT, which interestingly followed the lowest to the highest solubility of these solvents in water. The sequential cooling step showed the structures recovering at different levels and rates. More pronounced recovery occurred at the first minutes and slowed undergoing cooling as can be seen by $G^{\prime}$ values (Fig. 7a, c and e). However, a clear effect of oil polarity and chemical composition affecting the aggregation of sterols molecules in an oleogel system was observed and again, MCT and SFO showed to be more sensitive to temperature than HEX.

The time-dependent response of oleogels was investigated by frequency sweep which is exhibited in Fig. 7b, d and f. Overall, systems showed highest $G^{\prime}$ values compared to $G^{\prime \prime}$ values and frequency-independent $G^{\prime}$ which is characteristic of gels. Regarding solvent type effect, BG100 and BG80L20 formulations produced with HEX or SFO exhibited similar $G^{\prime}$ values with no conspicuous difference between them. These results indicate that until $20 \mathrm{wt} \%$ replacement of BG by L is feasible to produce SFO and HEX oleogels with similar elastic properties, which did not occur for MCT oleogels. The further increase of lecithin concentration, BG60L40, led to a notable reduction of elastic moduli and higher frequency dependence for all samples and to a higher extent for MCT.

Moreover, upon lecithin addition, the systems did not show instantaneous recovery of oleogel structure after application of shear and thermal events. The $G^{\prime}$ at $t=0$ (Fig. 7a, c and e) was different from initial $G^{\prime}$ in frequency sweep (Fig. $7 \mathrm{~b}, \mathrm{~d}$ and f) after the rheological measurements under non-isothermal conditions since phytosterol and sterol-ester based oleogels exhibit a gradual sol-gel transition dependent on the gelator concentration as reported previously (Bot \& Agterof, 2006; Matheson, Koutsos, et al., 2017). The time required to this event was considerably longer for $10 \mathrm{wt} \%$ gel than $20 \mathrm{wt} \%$ gel $(\sim 2500$ and $\sim 100 \mathrm{~s}$, respectively) (Matheson, Koutsos, et al., 2017). Usually the hierarchical organization takes time, because initially occurs the formation of fibrils and afterwards the aggregation of fibrils into highly ordered structures. Furthermore, there is the cumulative effect of lecithin presence, which promotes the delaying of network formation as seen from thermal analysis, which also contributed for increasing the time required for gelation.

\subsubsection{Large deformation}

The hardness, firmness, ductility and brittleness are important mechanical attributes which can define the potential industrial applicability of the oleogels in food, cosmetics or pharmaceutical products (Tavernier et al., 2017). A representative stress-strain curve for oleogels produced with HEX is shown in Fig. 8a. Fracture occurred at slightly lower strain values for BG100 (solid line) and the stress associated with rupture was lower and peak was broadening as $\mathrm{L}$ was included to form BG80L20 and BG60L40 (dashed and dotted), indicating that lecithin decreases the hardness of oleogel. There was a tendency that harder gels were produced with SFO, which was remarkable mainly at the highest lecithin content in the mixture (BG60L40) (Fig. 8b). Oleogels with no improvement in mechanical properties were produced upon lecithin addition and which resulted in BG reduction, since the phospholipid in the added amounts was not expected to directly contribute to gel strength.

Fracture strain (Fig. 8c) or maximum deformation before the rupture did not show a dependency on lecithin addition, however solvent type showed that SFO oleogels tend to be more ductile. For MCT and HEX oleogels, at highest phospholipid concentration (BG60L40), Young's modulus decreased meaning that structures became less stiff (Fig. 8d). On the other hand, SFO oleogels showed higher values of Young's modulus in this condition. In general, SFO oleogels produced harder and stiffer oleogels compared to the HEX and MCT even upon lecithin addition.

There was no direct relationship between the elastic modulus $G^{\prime}$ from rheological measurements and the hardness index. The explanation for these differences can be associated to a number of factors as the deformation magnitude (small or large), type of mechanical forces used in the measurements (shear or compression) and storage time of the samples. The rheological measurements were done after temperature sweep (with annealing set), while the large deformation measurements presented here were done at least after 7 days of storage at room temperature. The DSC measurements also confirmed this hypothesis since it was demonstrated that storage time played a key role in hybrid lecithin oleogels formation. Thus, harder fresh oleogels were produced with HEX, but after ageing period, SFO oleogels showed improved mechanical properties. In addition, for small deformation, much lower stress level is placed on the samples (below the yield stress), while for large deformation measurements high levels of strain is applied or samples are tested beyond the point of failure (Mohsenin \& Mittal, 1977). Both small and large deformation measurements are important, as the former elucidate the fundamental properties of the systems and the latter can give an indication of how the oleogel might behave during high stress or shear conditions simulating food processing and consumption (mastication, peristaltic movements) (Zetzl et al., 2014). At last different kind of applied forces (compression or shear) can give complementary information about the gels and their potential application, since the materials can respond in a different way depending on the force direction.

\section{Discussion}

Individually, neither $\beta$-sitosterol nor $\gamma$-oryzanol can be considered efficient oleogelators. However when combined, they may form doublewalled hollow tubules due to the establishment of $\beta$-sitosterol: $\gamma$-oryzanol complex by intermolecular hydrogen bonding, thus forming a continuous three-dimensional network (Bot \& Agterof, 2006; Rogers et al., 2010; Bot et al., 2012; Alhasawi \& Rogers, 2013). The probable growth mechanism of these structures would be the formation of helical ribbon from $\beta$-sitosterol and $\gamma$-oryzanol molecules, forming curved onedimensional aggregates that expand the wall along the central axis and align their androsterol groups (Bot et al., 2008; Laredo, Barbut, \& 


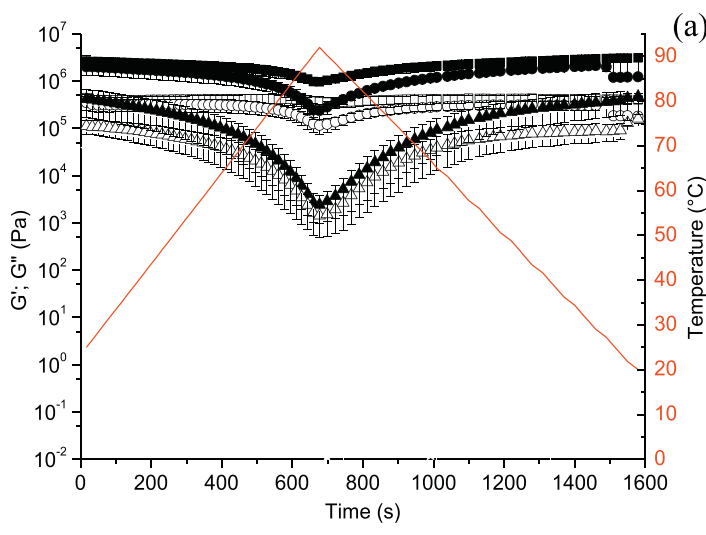

(a)
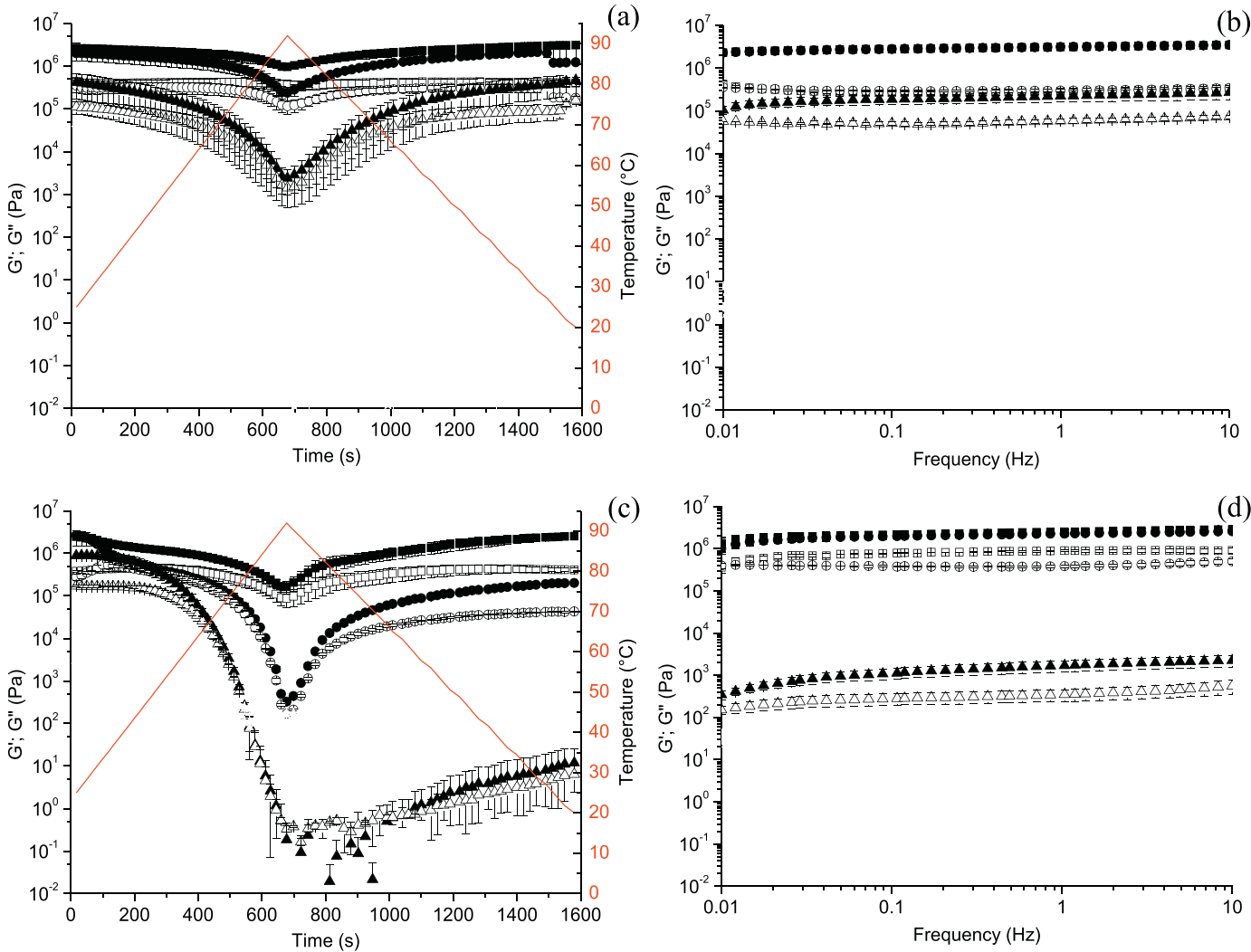

(c)
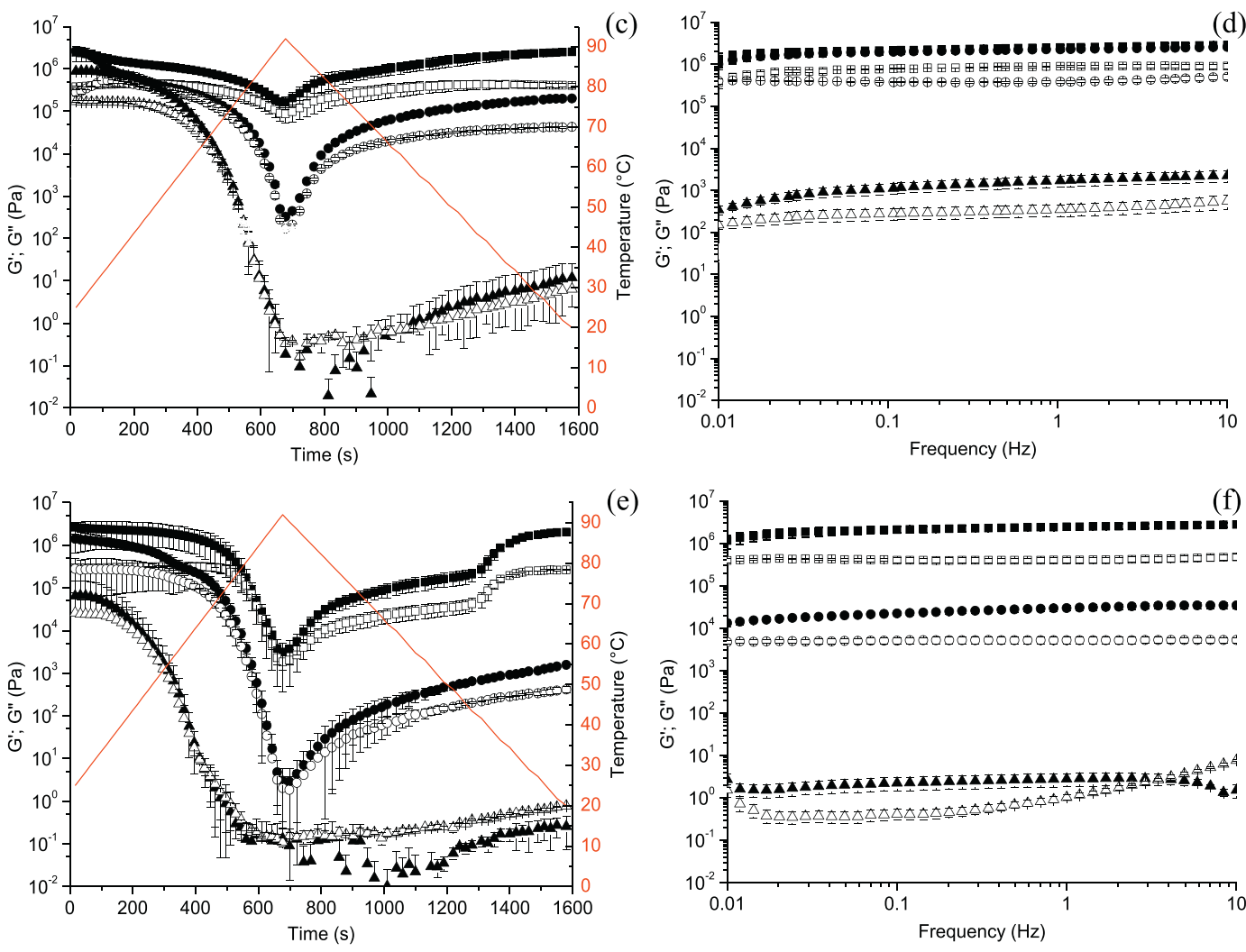

(e)

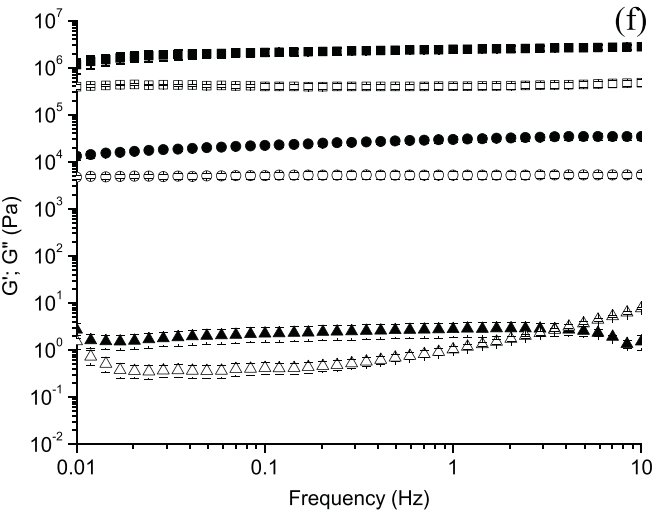

b)

(d)

\section{.}



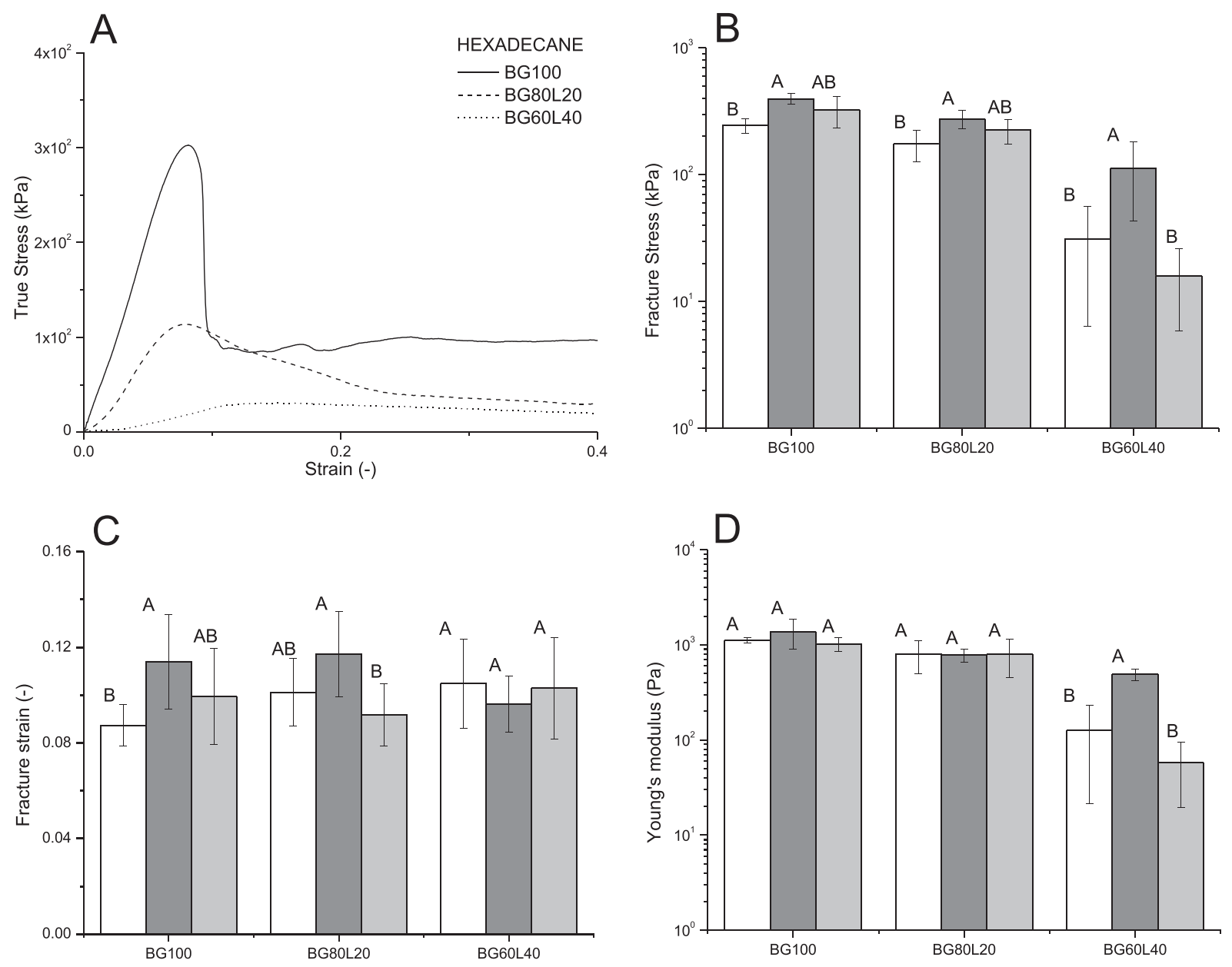

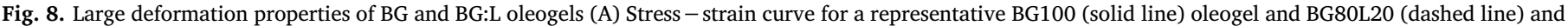

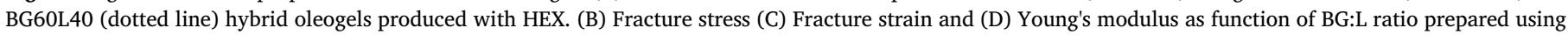

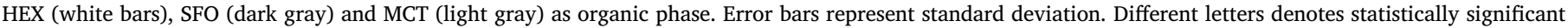
differences $(p<0.05)$ among the solvents for the same BG:L ratio.

the possible interactions with co-oleogelators might affect the behavior of host oleogelators self-assembly. Lecithin combined with sucrose esters (Bin Sintang et al., 2017) or $\alpha$-tocopherol (Nikiforidis \& Scholten, 2014) showed a favourable self-assembling due to minimization of the hydrophilic head group-solvent interactions. In these cases, a synergistic interaction was reported combining the main oleogelator with lecithin.

The phospholipid affected the kinetic of hybrid oleogels self-assembly. Longer time to achieve gelation was required for the formulations with phospholipid, as observed by DSC and non-isothermal rheological measurements. Therefore, in the presence of lecithin, instead of hydrogen bonding among $\beta$-sitosterol and $\gamma$-oryzanol molecules stack on top of each other at the ring moiety forming helical ribbon fibrils, a possible preferential linkage to the polar head group of phospholipids might be a possible explanation. A further molecular characterization on the self-assembly is necessary for better understanding of the interaction among B, G and L.

\section{Conclusions}

Different analyses were performed to understand the impact of incorporating lecithin into structured systems produced with a binary mixture of $\beta$-sitosterol (B) and $\gamma$-oryzanol (G). The replacement of $20 \mathrm{wt}$ $\%$ by lecithin did not have a significant impact on the large deformation mechanical properties; however, the further increase of lecithin in the mixture caused a remarkable reduction on mechanical strength of oleogel. Technological applications could be feasible in view of partial replacement of sterol(ester)s, and a further step in the use of non-purified lecithin could represent lower cost to oil structuring with similar mechanical properties.

Oleogels were produced using a healthy labelled mixture of oleogelators which provided different contributions to build the oleogel structure. Lecithin altered plant sterol(ester)s oleogel formation in different levels of organization. The phospholipid offered to BG system a kinetic dynamic feature considering that the organization of these molecules can be continuously recombining until reach a minimum energy state. Oleogels produced with SFO and lecithin addition formed harder and firmer systems compared to other solvents. Understanding multi component oleogelators allows tailoring gel properties varying the solvent quality and proportion of oleogelators in the mixture. The implication of changes in different levels of structure opens the opportunity to understand in which extent a co-oleogelator can impact in the system. The comprehension of lecithin behavior in combination with different host molecules can contribute in the control of gel response given the desired application contributing with the knowledge for ingredient engineering.

\section{Acknowledgements}

Paula Kiyomi Okuro thanks to CNPq (159180/2013-9) and São Paulo Research Foundation (FAPESP) grant \#2015/24912-4 and 2016/ 10277-8, for the PhD fellowship; Rosiane Lopes Cunha thanks CNPq 
(307168/2016-6) for the productivity grant. The authors also thank the Brazilian Synchrotron Light Laboratory (LNLS) for the opportunity to use SAXS1 (proposal number 20160277 and 20170297) beamline and Tsuno Rice Fine Chemicals Co., Ltd. for the donation of gamma oryzanol samples. We thank the access to equipment and assistance provided by the Laboratory of Process Engineering, Department of Food Engineering, Faculty of Food Engineering at the University of Campinas co-funded by FAPESP (96/08366-5, 2004/08517-3, 2006/03263-9, 2007/58017-5, 2011/06083-0).

\section{References}

Alhasawi, F. M., \& Rogers, M. A. (2013). Ternary phase diagram of $\beta$-Sitosterol- $\gamma$-oryzanol-canola oil. JAOCS, Journal of the American Oil Chemists' Society, 90(10), 1533-1540.

Bin Sintang, M. D., Danthine, S., Patel, A. R., Rimaux, T., Van De Walle, D., \& Dewettinck, K. (2017). Mixed surfactant systems of sucrose esters and lecithin as a synergistic approach for oil structuring. Journal of Colloid and Interface Science, 504, 387-396.

Blach, C., Gravelle, A. J., Peyronel, F., Weiss, J., Barbut, S., \& Marangoni, A. G. (2016). Revisiting the crystallization behavior of stearyl alcohol: Stearic acid (SO: SA) mixtures in edible oil. RSC Advances, 6(84), 81151-81163.

Bot, A., Den Adel, R., \& Roijers, E. C. (2008). Fibrils of $\gamma$-oryzanol $+\beta$-sitosterol in edible oil organogels. JAOCS, Journal of the American Oil Chemists' Society, 85(12), 1127-1134.

Bot, A., \& Agterof, W. G. M. (2006). Structuring of edible oils by mixtures of $\gamma$-oryzanol with $\beta$-sitosterol or related phytosterols. JAOCS, Journal of the American Oil Chemists' Society, 83(6), 513-521.

Bot, A., den Adel, R., Roijers, E. C., \& Regkos, C. (2009). Effect of sterol type on structure of tubules in sterol $+\gamma$-oryzanol-based organogels. Food Biophysics, 4(4), 266-272.

Bot, A., Gilbert, E. P., Bouwman, W. G., Sawalha, H., den Adel, R., Garamus, V. M., ... Flöter, E. (2012). Elucidation of density profile of self-assembled sitosterol + oryzanol tubules with small-angle neutron scattering. Faraday Discussions, 158, 223.

Bot, A., Veldhuizen, Y. S. J., den Adel, R., \& Roijers, E. C. (2009). Non-TAG structuring of edible oils and emulsions. Food Hydrocolloids, 23(4), 1184-1189.

Buerkle, L. E., \& Rowan, S. J. (2012). Supramolecular gels formed from multi-component low molecular weight species. Chemical Society Reviews, 41(18), 6089.

Gao, J., Wu, S., \& Rogers, M. A. (2012). Harnessing Hansen solubility parameters to predict organogel formation. Journal of Materials Chemistry, 22(25), 12651.

Gravelle, A. J., Blach, C., Weiss, J., Barbut, S., \& Marangoni, A. G. (2017). Structure and properties of an ethylcellulose and stearyl alcohol/stearic acid (EC/SO:SA) hybrid oleogelator system. European Journal of Lipid Science and Technology, 119(11), 1-11.

Gravelle, A. J., Davidovich-Pinhas, M., Zetzl, A. K., Barbut, S., \& Marangoni, A. G. (2016). Influence of solvent quality on the mechanical strength of ethylcellulose oleogels. Carbohydrate Polymers, 135, 169-179.

Han, L. J., Li, L., Zhao, L., Li, B., Liu, G. Q., Liu, X. O., \& De Wang, X. (2013). Rheological properties of organogels developed by sitosterol and lecithin. Food Research International, 53(1), 42-48.

Hunterlab (1996). Hunter Lab Color Scale. Insight on Color, 8(9).

Kumar, R., \& Katare, O. P. (2005). Lecithin organogels as a potential phospholipidstructured system for topical drug delivery: A review. AAPS PharmSciTech, 6(2),
E298-E310.

Lan, Y., \& Rogers, M. A. (2015). 12-Hydroxystearic acid SAFiNs in aliphatic diols - A molecular oddity. CrystEngComm, 17(42), 8031-8038.

Laredo, T., Barbut, S., \& Marangoni, A. G. (2011). Molecular interactions of polymer oleogelation. Soft Matter, 7(6), 2734.

Matheson, A. B., Dalkas, G., Gromov, A., Euston, S. R., \& Clegg, P. S. (2017). The development of phytosterol-lecithin mixed micelles and organogels. Food \& Function, 8(12), 4547-4554.

Matheson, A. B., Koutsos, V., Dalkas, G., Euston, S., \& Clegg, P. (2017). Microstructure of B-Sitosterol: $\gamma$-Oryzanol edible Organogels. Langmuir, 33(18), 4537-4542.

Mohsenin, N. N., \& Mittal, J. P. (1977). Use of rheological terms and correlation of compatible measurements in food texture research. Journal of Texture Studies, 8(4), $395-408$.

Nikiforidis, C. V., Gilbert, E. P., \& Scholten, E. (2015). Organogel formation via supramolecular assembly of oleic acid and sodium oleate. RSC Advances, 5(59), 47466-47475.

Nikiforidis, C. V., \& Scholten, E. (2014). Self-assemblies of lecithin and $\alpha$-tocopherol as gelators of lipid material. RSC Advances, 4(5), 2466-2473.

Patel, A. R. (2017). A colloidal gel perspective for understanding oleogelation. Current Opinion in Food Science, 15, 1-7.

Patel, A. R., \& Dewettinck, K. (2016). Edible oil structuring: An overview and recent updates. Food \& Function, 7(1), 20-29.

Pernetti, M., van Malssen, K., Kalnin, D., \& Flöter, E. (2007). Structuring edible oil with lecithin and sorbitan tri-stearate. Food Hydrocolloids, 21(5-6), 855-861.

Rogers, M. A., Bot, A., Lam, R. S. H., Pedersen, T., \& May, T. (2010). Multicomponent hollow tubules formed using phytosterol and $\gamma$-oryzanol-based compounds: An understanding of their molecular embrace. Journal of Physical Chemistry A, 114(32), 8278-8285.

Sawalha, H., Den Adel, R., Venema, P., Bot, A., Flöter, E., \& Van Der Linden, E. (2012). Organogel-emulsions with mixtures of $\beta$-sitosterol and $\gamma$-oryzanol: Influence of water activity and type of oil phase on gelling capability. Journal of Agricultural and Food Chemistry, 60(13), 3462-3470.

Sawalha, H., Venema, P., Bot, A., Flöter, E., Den Adel, R., \& Van Der Linden, E. (2015). The phase behavior of $\gamma$-Oryzanol and $\beta$-Sitosterol in edible oil. JAOCS. Journal of the American Oil Chemists' Society, 92(11-12), 1651-1659.

Tamura, T., \& Ichikawa, M. (1997). Effect of lecithin on organogel formation of 12-hydroxystearic acid. Journal of the American Oil Chemists' Society, 74(5), 491-495.

Tavernier, I., Doan, C. D., Van de Walle, D., Danthine, S., Rimaux, T., \& Dewettinck, K. (2017). Sequential crystallization of high and low melting waxes to improve oil structuring in wax-based oleogels. RSC Advances, 7(20), 12113-12125.

Terech, P., \& Weiss, R. G. (1997). Low molecular mass Gelators of organic liquids and the properties of their gels. Chemical Reviews, 97(8), 3133-3160.

Von Bonsdorff-nikander, A., Karjalainen, M., Rantanen, J., \& Christiansen, L. (2003). P hysical stability of a microcrystalline b-sitosterol suspension in oil. European Journal of Pharmaceutical Sciences, 19, 173-179.

Xu, Z., \& Godber, J. S. (1999). Purification and identification of components of $\gamma$-oryzanol in rice bran oil. Journal of Agricultural and Food Chemistry, 47(7), 2724-2728.

Yin, D., Liu, J., Geng, W., Zhang, B., \& Zhang, Q. (2015). Microencapsulation of hexadecane by surface-initiated atom transfer radical polymerization on a Pickering stabilizer. New Journal of Chemistry, 39(1), 85-89.

Zetzl, A. K., Gravelle, A. J., Kurylowicz, M., Dutcher, J., Barbut, S., \& Marangoni, A. G. (2014). Microstructure of ethylcellulose oleogels and its relationship to mechanical properties. Food Structure, 2(1-2), 27-40. 